\title{
Non-uniform Gradient Prescription for Precise Angular Measurements Using DTI
}

\author{
Nathan Yanasak ${ }^{1}$, Jerry D. Allison ${ }^{1}$, Qun Zhao ${ }^{2}$, Tom C.-C. Hu ${ }^{1}$, \\ and Krishnan Dhandapani ${ }^{3}$ \\ ${ }^{1}$ Department of Radiology, Medical College of Georgia, USA \\ ${ }^{2}$ Department of Physics, University of Georgia, USA \\ ${ }^{3}$ Deparment of Neurosurgery, Medical College of Georgia, USA \\ nyanasak@mcg.edu
}

\begin{abstract}
Diffusion Tensor Imaging (DTI) calculates a tensor for each voxel, representing the mean diffusive characteristics in volume-averaged tissue. Gradients that phase-encode spins according to the amount of their diffusion are usually applied uniformly over a sphere during a DTI procedure for minimal bias of tensor information. If prior knowledge of diffusion direction exists, the angular precision for determining the principle eigenvector of cylindrically-symmetric ("prolate") tensors can be improved by specifying gradients non-uniformly. Improvements in precision of $30-40 \%$ can be achieved using a restricted band of zenith angle values for gradient directions. Sensitivity to the a priori angular range of the principle eigenvector can be adjusted with the width of the band. Simulations and phantom data are in agreement; a preliminary validation is presented.
\end{abstract}

\section{Introduction}

The technique of diffusion tensor imaging (DTI) provides information about the anisotropy of diffusive water movement in tissue as well as the magnitude of diffusion. Clinical use of DTI has focused primarily on pathological changes as observed in the mean apparent diffusion coefficient (ADC) in a region of interest, or the fractional anisotropy (FA). Examples of this include the reduction of anisotropy from myelin destruction in sclerotic lesions [1, or the evolution of Wallerian degeneration following stroke [2].

Generally, no assumptions are made about the nature of tissue imaged with DTI. To measure tissue diffusivity precisely, gradients that create phase dispersion in proportion to diffusion along an axis are applied uniformly over a sphere 3. However, information concerning anisotropy of a set of ADC measurements is not uniformly distributed among those gradient directions. If some prior knowledge of the tissue directionality is given (e.g., corticospinal tracts), gradients can be applied non-uniformly to improve DTI metrics, leading to pathological specificity [4]. For example, Peng, et al. 5] demonstrated an optimization scheme that specified gradient directions for maximum sensitivity to FA if the tissue had prolate diffusion characteristics, where the ADC measurements form a "peanut"shaped spatial distribution. In general, other parameters such as b-value could

D. Metaxas et al. (Eds.): MICCAI 2008, Part I, LNCS 5241, pp. 866-873, 2008.

(C) Springer-Verlag Berlin Heidelberg 2008 
also be varied non-uniformly during acquisition. Considering the use of multiple diffusion weightings in techniques such as in high angular resolution diffusion imaging (HARDI, 6]), this approach would be critical; parametric optimization methods used in previous studies (e.g., [5], [7]) would provide an efficient framework to search for solutions.

For tissues exhibiting diffusion predominantly along one direction (e.g., white matter tracts), DTI reveals the principle axis of diffusion within an image voxel. By "connecting" these principle axes together, Diffusion Tensor Tractography has been employed to reconstruct tissue structure orientation. Recent applications include characterization of tumor infiltration of white matter [8, coronary muscle fiber remapping in heart failure 9, and diseases and trauma of the spinal cord 10]. As with studies involving ADC or FA, improvement of angular precision in measuring principle diffusion directions could increase clinical specificity. For example, improved tracking could set quantitative limits on normal white matter tract deviation in the presence of tumors, in the case of tissues such as spinal cord or brain stem.

In this study, we demonstrate that a non-uniform set of gradient directions can lead to improved angular precision of the principle eigenvector for a prolate-like ADC distribution. Because of the azimuthal symmetry of a prolate distribution in spherical coordinates, gradient directions from a uniform distribution were compressed along the zenith angle to identify angles for optimal sensitivity to angular precision. It was anticipated that the optimal sensitivity would correspond to angular ranges, or "bands", that balance two characteristics: 1) curvature of the ADC distribution that changes quickly as a function of angle, and 2) distance away from the poles of the distribution, where signal attenuation may contribute more error proportionally to the ADC distribution. Using angular bands of different widths, the sensitivity of angular precision to uncertainty in the principle eigenvalue of the prolate distribution was characterized. Simulations were verified using phantom data, and improvement in angular precision was validated using human brain DTI images.

\section{Method}

\section{$2.1 \quad$ Simulation}

DTI data were simulated using Matlab (The Mathworks, Natwick, MA), and they were fit to a tensor to determine the angular dispersion of the principle eigenvalue for a set of noisy measurements. Water diffusivity was assumed to follow a prolate distribution with the following eigenvalues: $\lambda=[2.4,0.65$, $0.65] \times 10^{-3} \mathrm{~mm}^{2} / \mathrm{sec}(\mathrm{FA}=0.68)$. Beginning with gradient directions specified for a GE clinical scanner, new gradient directions for diffusion-weighting were determined by compressing the original directions into a limited range of zenith angles, assuming that the largest eigenvalue points along the $\hat{z}$-axis.

Given the prolate diffusion distribution and a non-diffusion-weighted signal intensity for water, diffusion-weighted intensities were calculated for each prescribed gradient direction. For each gradient direction, the diffusion-weighted 
intensity was duplicated into multiple samples and Fourier transformed. Gaussian noise was added to complex data before inverse Fourier transform, yielding multiple, unique samples of the prolate distribution as a proxy to multiple voxels within an ROI. Tensors were fit to each sample using a weighted least-squares method [11. A dot product between the principle diffusion axis for each tensor and the prolate distribution axis was employed to find the angle between the two vectors $(\eta)$, characterizing angular precision. Angular dispersion, $\sigma_{\eta}$, was defined as the angle at which $68 \%$ of the measurements were equal or smaller.

\subsection{Phantom}

A phantom with arrays of glass capillaries (20 $\mu \mathrm{m}$ i.d.) was used to verify simulation results. The phantom consisted of a $5 \times 5 \times 5$ in. ${ }^{3}$ water-filled container, with a $1 \times 1 \times 0.3$ in. $^{3}$ region of capillary arrays. Characteristics of these arrays have been reported previously 12 .

DTI images were acquired using a GE Excite HDx 3T MRI scanner (GE Medical Systems, Milwaukee, WI), with an eight-channel head coil. The following DW-EPI protocol was used: 3 slices; TR/TE $=3000 / 80$ msec; $128 \times 128$ matrix; $16 \mathrm{~cm} \mathrm{FOV} ; 4 \mathrm{~mm}$ slice thickness; $1.5 \mathrm{~mm}$ gap; ASSET factor $=2 ; \mathrm{b}=1000 \mathrm{~mm}^{-2}$; $3 \mathrm{~b}=0$ images. The image plane of the second slice was aligned in parallel with the capillary arrays, with the largest eigenvalue in the phantom parallel to the $\hat{y}$-axis. Gradient directions and number of directions were specified as per the simulation, switching the $\hat{z}$ and $\hat{y}$ coordinates for alignment of the long axis of the prolate. Capillary ROIs were chosen for each image series, resulting in $\approx$ $280-325$ voxels. The $\mathrm{SNR}$ values of the $\mathrm{b}=0$ images were $\approx 38$ within the ROIs. Noise characteristics and number of sampled measurements in the simulations were chosen to match these values.

When comparing angular dispersion, Bartlett's test was used to verify a significant difference between one or more gradient direction schemes. Once a significant difference was found between multiple schemes, two-sample F-tests were used to identify significance of differences between pairs. Using a Bonferroni correction to account for multiple comparisons, $\alpha$ was lowered to 0.005. For 300 measurements per dispersion, values of $\mathrm{F} \geq 1.34$ were considered to be significant.

\subsection{Validation}

A previously-acquired clinical DTI scan was used for validation of the phantom and simulation results. The following DW-EPI protocol was used: 22 slices; $\mathrm{TR} / \mathrm{TE}=6000 / 78 \mathrm{msec} ; 128 \times 128$ matrix; $24 \mathrm{~cm}$ FOV $; 5 \mathrm{~mm}$ slice thickness; $1.5 \mathrm{~mm}$ gap; ASSET factor $=2 ; 25$ directions; $b=1000 \mathrm{sec} \mathrm{mm}^{-2} ; 2 \mathrm{~b}=0$ images. Six small ROIs containing neural fibers from the corpus callosum were selected. Within each ROI, the angular dispersion of the principle eigenvector around their mean was less than $5^{\circ}$. The ROIs, composed of 91 voxels total, were defined using an atlas to interpret the FA map 13 . 

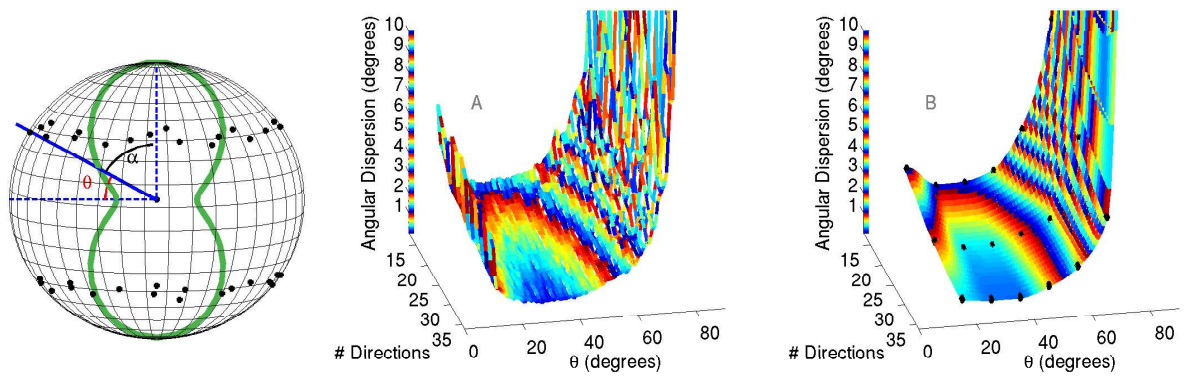

Fig. 1. Angular precision for measuring the eigenvector of principle diffusion for a prolate ADC distribution (green).The gradient directions, specified in a ring at elevation angle $\theta$, are shown as black dots on a unit sphere. The zenith angle, $\alpha$, is also shown. Panel A: simulated precision; Panel B: measured precision. One color cycle (e.g., red to red) $=1^{\circ}$.

Using the mean principle eigenvector in each ROI determined from the 25direction image series, two subsets of directions were chosen for recalculation of the tensor. The first subset, selected for higher angular precision, consisted of directions within a band of elevation angles $15^{\circ}<\theta<45^{\circ}$. The elevation angle is given as the complementary angle to the zenith angle, $\alpha$, in spherical coordinates (see Figure 1). The second subset, selected for ordinary angular precision, used a set of directions distributed evenly over the surface of a sphere. The number of directions in the second subset was the same as the first subset (10-12), allowing a direct comparison of the angular precision of the principle eigenvalue between both sets of directions. For each direction set, the angular difference of each principle eigenvector from the mean eigenvector was determined within each ROI, and the composite angular precision was determined using all of these differences. The significance of differences in the angular precision was characterized using a two-sample $\mathrm{F}$-test $(\mathrm{F} \geq 1.42$ corresponds to $\mathrm{p}<0.05$ for 91 degrees of freedom). The choice of a smaller number of directions in the second subset, from the 25 directions distributed evenly over a sphere, resulted in a distribution that was approximately homogeneous. As a result, this procedure may be considered to be only a partial, preliminary validation.

\section{Results}

Simulations examined the sensitivity of angular precision to elevation angle, given a prolate distribution with the thin "waist" defined by $0^{\circ}$ elevation. Gradient directions were compressed into a band of angles $10^{\circ}$ wide, with a mean elevation angle of $\theta$ as shown in Figure 1. Panel A in Figure 1 shows the angular dispersion as a function of $\theta$ and the number of gradient directions. For the FA value in this simulation and for a DTI sequence having 36 gradient directions, the optimal elevation angle is $\theta=28.7^{\circ}$, resulting in a dispersion of the measured principle eigenvector $\sigma_{\eta}=1.1^{\circ}$. Phantom measurements, shown as black 

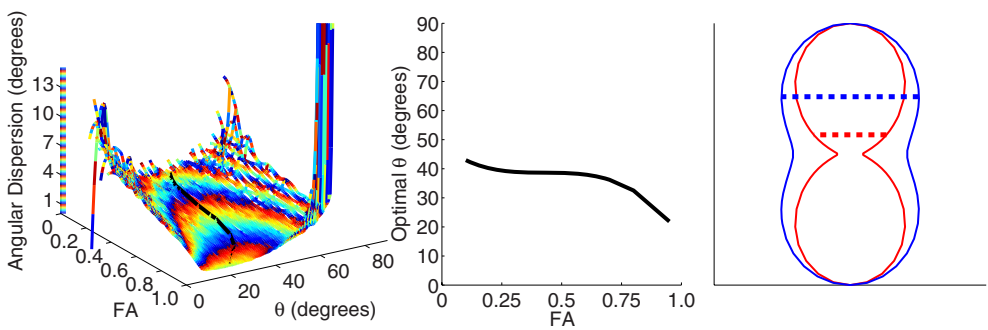

Fig. 2. Angular precision for measuring the eigenvector of principle diffusion for a prolate $\mathrm{ADC}$ distribution, as a function of prolate FA and elevation angle, $0^{\circ} \leq \theta \leq 70^{\circ}$. The optimal elevation angle is also shown in black, as a function of FA. One color cycle $=1^{\circ}$. Right Panel: Dashed lines show the region of optimal $\theta$, for ADC distributions with $\mathrm{FA}=0.5$ (blue) and $\mathrm{FA}=0.9$ (red).
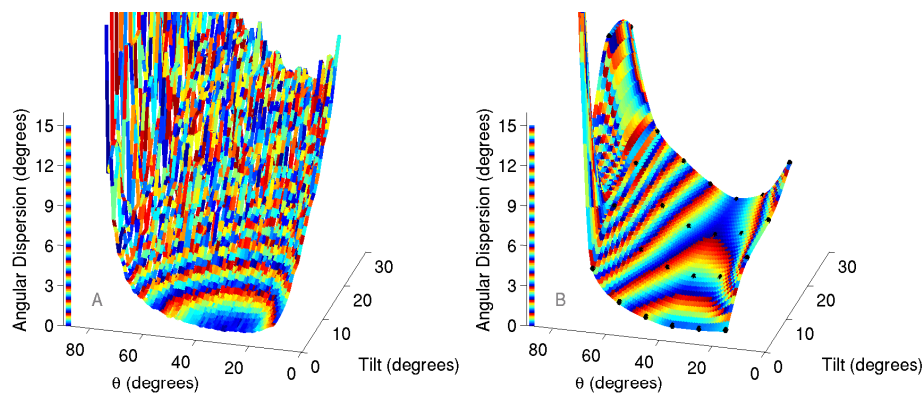

Fig. 3. Angular precision for measuring the eigenvector of principle diffusion for a prolate ADC distribution, with gradient directions specified in Figure 1. In this case, the principle eigenvector is tilted away from the poles of the sphere. Panel A: simulated precision; Panel B: measured precision. One color cycle $=1^{\circ}$.

points on an interpolated surface in Panel B of Figure 1, qualitatively match with simulations. Data for a DTI sequence having 36 directions, acquired at $10^{\circ}$ increments in the elevation angle, gives an optimal elevation angle of $\theta \approx 35^{\circ}$, with an angular dispersion of $1.2^{\circ}$.

The dependence of optimal elevation angle on FA is shown in Figure 2. The minimum angular dispersion for each value of FA is shown as a black line in the left and right panels, indicating the optimal elevation angle. Up to a value of $\mathrm{FA}=0.8$ or so, the optimal elevation angle is fairly consistent at $\sim 30^{\circ}-40^{\circ}$. As shown in the right panel of Figure 2, the optimal angle corresponds to areas where the contours of the ADC distribution are changing rapidly, as expected.

Gradient specification within a thin angular band yields improved angular precision if the prolate direction is known. In practice, the direction is known coarsely, and the precision will depend on how far the prolate is tilted with respect to the band. Figure 3 compares angular precision from simulations (Panel A) and phantom data (Panel B) for a DTI sequence having 36 directions, as a 
function of $\theta$ and tilt of the prolate eigenvector. Once again, both data and simulation qualitatively compare favorably, with some difference in behavior at higher tilt angles. At an elevation angle of $35^{\circ}$, simulated angular dispersion increases by $\Delta \sigma_{\eta}=11.5^{\circ}$ as the prolate tilts from a zenith angle of $0^{\circ}$ to $30^{\circ}$. For measured data, the precision degrades by $\Delta \sigma_{\eta}=1.9^{\circ}$. Simulations appear to deviate from measurements along the surface near the optimal angle in Figure 3 . as the tilt angle increases. The origin of this difference is under investigation.

Figure 4 shows four different gradient prescriptions for comparison, using bands of different angular width to balance tilt effects. All bands were centered at an elevation angle of $30^{\circ}$, where angular precision is near optimal. Simulations demonstrate that, as the band width increases, the immunity to tilt also increases while retaining low angular dispersion. At this FA value (0.68), a band width of $36^{\circ}$ is the best compromise between minimal angular dispersion and tilting effects out to $30^{\circ}$. Increasing the width continues to improve the immunity to tilt but decreases the angular precision (as in Figure 30). This trend is also present in phantom data, although angular dispersion increases more slowly as a function of tilt angle. Unlike the simulation, the widest band $\left(58^{\circ}\right)$ does not show a significant increase in mean dispersion for all tilt angles. Clearly, for a band somewhat thicker than $58^{\circ}$, the gradient directions will approximate spherically-homogeneous sampling, and curve "D" in the right panel of Figure 4 will conform to the curve for the spherical distribution of gradient directions as per the simulations.

Bartlett's test identified significant differences between precision at all four measured tilt angles $(\mathrm{p}<0.001)$. Using two-sample F-tests corrected via Bonferroni for 10 different comparisons at each tilt angle, groups of precision values that were not significant to $\mathrm{p}<0.005$ are indicated in Figure 4 within red circles. It should be noted that without the Bonferroni correction, precision for curves "C" and "D" at $10^{\circ}$ tilt are significantly different from all others $(\mathrm{p}<0.05)$. Considering the multiple-comparison correction to be conservative, this suggests marginal significance for the phantom results at these tilt angle, although more precise measurements are required for confirmation.

The importance of the angular thickness of the band for scheme $\mathrm{C}$ in Figure 4 can be understood using the sensitivity of angular dispersion to elevation angle shown in Figure 1 In the angular range of the band $\left(\theta=15^{\circ}-45^{\circ}\right)$, the distribution of angular dispersion is fairly flat, although the angular dispersion increases fairly quickly for larger and smaller elevation angles. By confining the band to this range, the "trough" in the angular dispersion surface is utilized, while tilt becomes less influential due to the band width.

Expectedly, the improvement of angular precision scales with SNR according to simulation. From Figure 4, use of scheme $\mathrm{C}$ results in an improvement in the precision $\sim 30-40 \%$ at maximum. Phantom data supports a similar improvement.

Figure 5 shows the angular distribution in an ROI of the principle eigenvectors for a human brain scan, for a homogeneous distribution of gradient directions and for gradients within an angular band $\left(\theta=15^{\circ}-45^{\circ}\right)$. The angular precision values using these 10-12 gradient directions are $\sigma_{\eta}=6.5^{\circ}$ and $3.8^{\circ}$, respectively. 

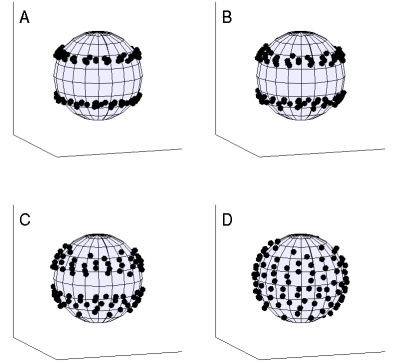
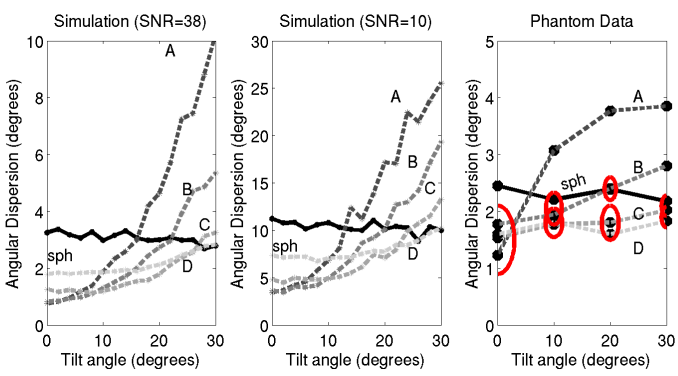

Fig. 4. Angular precision as a function of tilt angle, for four different gradient direction schemes. Note that the north and south hemispheres are mirrored. A: $10^{\circ}$ thick ring; B: $18^{\circ}$ thick band; C: $36^{\circ}$ thick band; D: $58^{\circ}$ thick band. The black line indicates the angular precision of a spherically-homogeneous set of gradient directions. Red circles indicate values of precision that are insignificantly different ( $\mathrm{p}>0.005$, Bonferroni-corrected).
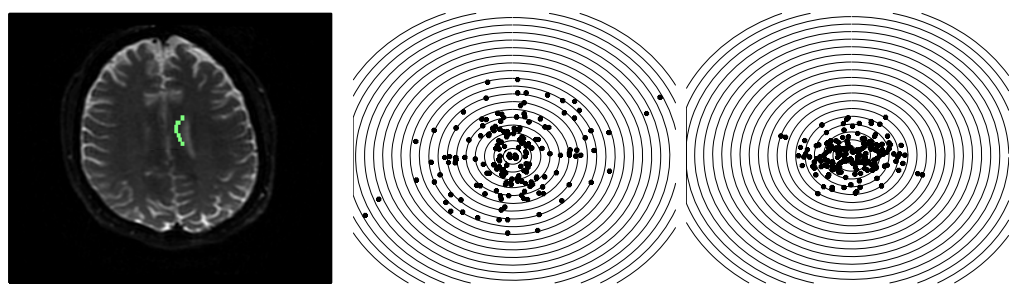

Fig. 5. Angular precision using data from the corpus callosum (green ROIs, left panel). Center panel: deviation of principle eigenvalues from $0^{\circ}$, for uniform distribution of gradient directions. Right panel: deviation of principle eigenvalues, for gradient directions within an angular band. Each ring represents $1^{\circ}$.

For comparison, the angular precision from analysis of the 25-direction scheme is $3.1^{\circ}$. The F-test verifies that the $10-12$ gradient schemes are significantly different $(\mathrm{F}=2.84 ; \mathrm{p}<0.001)$, indicating an improvement of $\Delta \sigma_{\eta}=42 \%$ using the distribution within an angular band, and consistent with Figure 4 . A slight significance between the 10-12 direction ring scheme and the 25-direction scheme is indicated $(\mathrm{F}=1.53 ; \mathrm{p}=0.04)$. This observation affirms previous conclusions that increasing the numbers of gradient directions is generally better, although the gained improvement in precision is only $18 \%$.

\section{Discussion}

If tissue geometry is prolate and the direction of the principle eigenvector is known to within $\leq 30^{\circ}$, we have shown that angular precision in determining the principle eigenvector can be optimized using a non-uniform gradient direction prescription. Most of the information describing the orientation of the prolate 
tissue is contained within a band of elevation angles $\theta \approx 15^{\circ}-45^{\circ}$ degrees. Simulations and phantom data show that the use of gradients within a band can give better precision than using the same number over a sphere. This improvement could be used to improve clinical specificity; additionally, the number of directions could be decreased from typical clinical protocols to achieve similar precision at a time savings. While elevation of the band determines the sensitivity of gradients to angular precision, band width provides some immunity to uncertainty in the tilt of the prolate to the scan plane. A band width of $\sim 30^{\circ}-50^{\circ}$ provides a reasonable amount of tilt immunity and angular precision for a large range of FA values, based on Figure 2. This preliminary study does not address whether the precision and accuracy of FA degrades while angular precision is optimized. FA accuracy could be improved by combining gradient bands with a small number of directions near the poles. Further validation of improvement in the angular precision in DTI using biological samples is warranted.

\section{References}

1. Filippi, M., et al.: Diffusion tensor magnetic resonance imaging in multiple sclerosis. Neurology 56, 304-311 (2001)

2. Gupta, R.K., et al.: Focal wallerian degeneration of the corpus callosum in large middle cerebral artery stroke: Serial diffusion tensor imaging. Journal of Magnetic Resonance Imaging 24, 549-555 (2006)

3. Papadakis, N.G., et al.: A comparative study of acquisition schemes for diffusion tensor imaging using MRI. Journal of Magnetic Resonance 137, 67-82 (1999)

4. Schmierer, K., et al.: Diffusion tensor imaging of post mortem multiple sclerosis brain. NeuroImage 35, 467-477 (2007)

5. Peng, H., Arfanakis, K.: Diffusion tensor encoding schemes optimized for white matter fibers with selected orientations. Magnetic Resonance Imaging 25, 147-153 (2007)

6. Frank, L.: Characterization of anisotropy in high angular resolution dw MRI. Magnetic Resonance in Medicine 47, 1083-1099 (2002)

7. Alexander, D.C.: Axon radius measurements in vivo from diffusion MRI: a feasibility study. In: Proceedings of the 11th ICCV, pp. 1-8 (2007)

8. Yu, C.S., et al.: Diffusion tensor tractography in patients with cerebral tumors: A helpful technique for neurosurgical planning and postoperative assessment. European Journal of Radiology 56, 197-204 (2005)

9. Helm, P.A., et al.: Evidence of structural remodeling in the dyssynchronous failing heart. Circulation Research 98, 125-132 (2006)

10. Vargas, M.I., et al.: Clinical applications of diffusion tensor tractography of the spinal cord. Neuroradiology 50, 25-29 (2008)

11. Salvador, R.G., et al.: Formal characterization and extension of the linearized diffusion tensor model. Human Brain Mapping 24, 144-155 (2005)

12. Yanasak, N., Allison, J.D.: Use of capillaries in the construction of an MRI phantom for assessment of diffusion tensor imaging: Demonstration of performance. Magnetic Resonance Imaging 24, 1349-1361 (2006)

13. Mori, S., Wakana, S., Nagae-Poetscher, L.M., Van Zijl, P.C.M.: MRI Atlas of Human White Matter. Elsevier, Amsterdam (2005) 